\title{
On Square Sum Labeling of Two Families of Petersen Graphs
}

\author{
Zhiqiang Zhang $\mathbb{D},{ }^{1}$ Muhammad Naeem $\mathbb{D}^{2}$, Abeera Tariq $\mathbb{D}^{2}$, and Weidong Zhao $\mathbb{D}^{1}$ \\ ${ }^{1}$ School of Computer Science, Chengdu University, Chengdu, China \\ ${ }^{2}$ Department of Mathematics and Statistics, Institute of Southern Punjab, Multan, Pakistan
}

Correspondence should be addressed to Muhammad Naeem; naeempkn@gmail.com

Received 14 December 2021; Accepted 25 January 2022; Published 18 February 2022

Academic Editor: Gohar Ali

Copyright (c) 2022 Zhiqiang Zhang et al. This is an open access article distributed under the Creative Commons Attribution License, which permits unrestricted use, distribution, and reproduction in any medium, provided the original work is properly cited.

A labeling on a graph $G$ with $n$ vertices and $m$ edges is called square sum if there exists a bijection $f: V(G) \longrightarrow\{0,1,2,3, \ldots, n-1\}$ such that the function $f^{*}: E(G) \longrightarrow N$ defined by $f^{*}(s t)=(f(s))^{2}+(f(t))^{2}$, for all st $\in E(G)$, is injective. A graph $G$ having a square sum labeling is called square sum. In this study, we have investigated the square sum labeling of generalized Petersen graph and double generalized Petersen graph.

\section{Introduction}

In [1], Germina et al. derived square sum labeling for basic graphs such as trees and cycles. Among various labeling methods that have evolved since 1960, one of the significant labeling methods is square sum. The vast history of the sum of squares of the integers motivated them to study and define this labeling in the particular graphs, and they named it square sum graphs. Many problems in real life and abstract thoughts, which are based on several conditions and with practical implications, can be studied by understanding modeling those problems by graphs, and this problem is one of the uses of graphs in handling and attempting to solve a problem of number theory. A graph which admits square sum labeling is called a square sum graph. In [1], Germina et al. proved that the complete graph $K_{n}$ is square sum iff $n \leq 7$ and the other graphs which they proved to be square sum are trees, unicyclic graphs, $m C_{n}$, and cycles' chord; the graphs obtained by connected two copies of cycle $C_{n}$ passes through the path $P_{k}$, a path union of $k$ copies of $C_{n}$, and the path is $P_{2}$.

Let $G$ be a graph and $p$ and $q$ be its vertices and edges, respectively. A labeling on the vertices $G$ can also be called a function with domain $V(G)$. An injective function with domain $V(G)$ and range $\{0,1,2, \ldots, p-1\}$ is called square sum labeling if the induced function of $f$, namely, the function $f^{*}$ with domain $E(G)$ and defined as $f^{*}(s t)=(f(s))^{2}+(f(t))^{2}$, for all st $\in E(G)$, is also injective. The square sum graphs are the graphs having a square sum labeling. We would also like to mention that not all graphs are square sum graphs. For instance, the complete graphs $K_{n}$, for $n \geq 6$, are not square sum graphs.

In number theory form, the statement that "a number $n$ can be written as a sum of two squares integers" is equivalent to "every prime of the form $(4 k+3)$ occurs an even number of times in the prime factorization of $n$." The edge labels in square sum labeling is of the form $n=a^{2}+b^{2}$. So, a number $n$ cannot be an edge label of a square sum graph if in its prime factorization a prime of the form $(4 k+3)$ (if it exists) occurs an odd number of times. Seoud and Al-Harere [2] proved many necessary conditions of square sum graphs, and they showed that $2 C_{n}, P_{2 n}$, and $C_{2 n}$ are square sum graphs. In [3], Huilgol and Sriram prove that if $G_{1}$ and $G_{2}$ are square sum, then $G_{1} \cup G_{2} \cup G_{3}$ is also square sum, where $G_{3}$ is a set of isolated vertices, for more literature review (see [2-5]). 


\section{Main Results}

In this section, we will present our results of this article.

Theorem 1. The generalized Petersen graph $P(n, k)$ is a square sum graph, for all $n \geq 5$.

Proof. Suppose $G \cong P(n, k)$ be the generalized Petersen graph, where $2 \leq k \leq\lfloor n / 2\rfloor$. We shall show that $G$ is square sum graph by defining a square sum labeling on $G$. For this purpose, we shall consider two cases one is when $n$ is even and other when $n$ is odd.

So, suppose $f: V(G) \longrightarrow\{0,1, \ldots,|V(G)-1|\}$ be a function defined as follows.

Case 1. When $n$ is even.

The labeling of $\left(y_{i}\right)$ for $2 \leq i \leq(n / 2)-1$ is

$$
f\left(y_{i}\right)=4 i-2 .
$$

The labeling of $f\left(y_{0}\right)$ and $f\left(y_{1}\right)$ is fixed:

$$
\begin{aligned}
& f\left(y_{0}\right)=1, \\
& f\left(y_{1}\right)=2 .
\end{aligned}
$$

The labeling of $\left(y_{i}\right)$ for $(n / 2)+1 \leq i \leq n-1$ is

$$
\begin{aligned}
& f\left(y_{i}\right)=2 n-3-4\left(i-\frac{n}{2}-1\right), \\
& f\left(y_{i}\right)=2 n-1, \quad i=\frac{n}{2} .
\end{aligned}
$$

The labeling of $f\left(x_{0}\right)$ and $f\left(x_{1}\right)$ is fixed:

$$
\begin{aligned}
& f\left(x_{0}\right)=0, \\
& f\left(x_{1}\right)=3 .
\end{aligned}
$$

The labeling of vertices $x_{i}$ for $2 \leq i \leq(n / 2)-1$ is

$$
\begin{aligned}
& f\left(x_{i}\right)=4 i-1, \\
& f\left(x_{i}\right)=2 n-2, \quad i=\frac{n}{2} .
\end{aligned}
$$

The labeling of $x_{i}$ for $(n / 2)+1 \leq i \leq n-1$ is

$$
f\left(x_{i}\right)=2 n-4-4\left(i-\frac{n}{2}-1\right) .
$$

The labeling of $x_{i}$ is

$$
\begin{aligned}
f\left(x_{i}\right) & =2 n+\frac{4 n}{2}-4 i \\
& =2 n+2 n-4 i \\
& =4 n-4 i .
\end{aligned}
$$

This labeling is explained in Figure 1.

Now, we shall discuss the weights of the edges induced by the above labeling of vertices of $G$. The weight of the edge $u v \in E(G)$ will be denoted as $f^{*}(u v)=(f(u))^{2}+(f(v))^{2}$. So, the induced labeling of the edge $x_{i} y_{i}$ for $0 \leq i \leq n-1$ is given as follows.

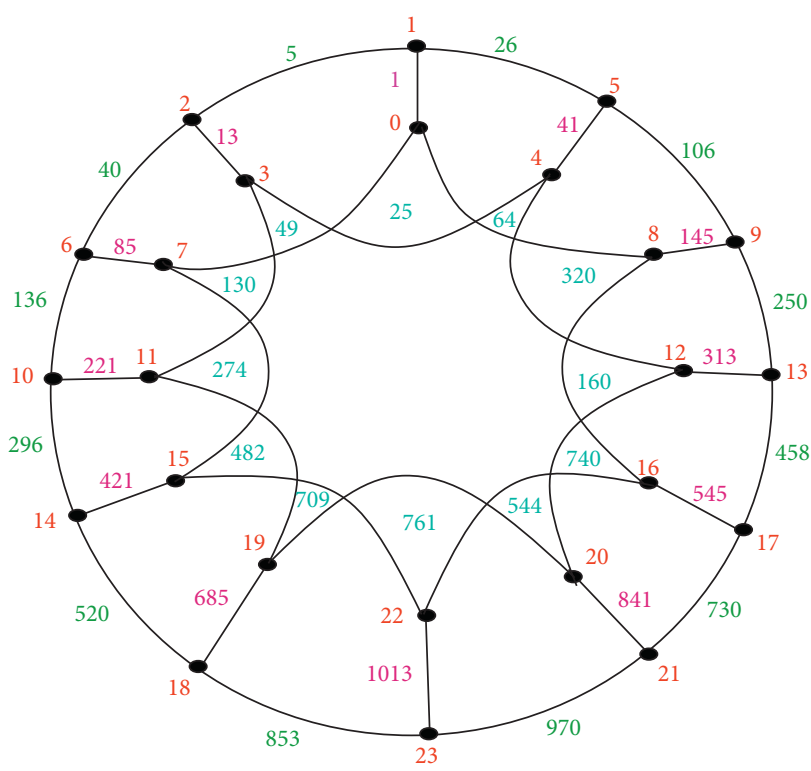

Figure 1: Square sum labeling of Petersen graph $P[12,2]$.

The labeling of $x_{i} y_{i}$, for $2 \leq i \leq(n / 2)-1$, is

$$
\begin{aligned}
f^{*}\left(x_{i} y_{i}\right) & =f\left(x_{i}\right)^{2}+f\left(y_{i}\right)^{2} ; \quad 2 \leq i \leq \frac{n}{2}-1, \\
& =(4 i-1)^{2}+(4 i-2)^{2} \\
& =32 i^{2}-24 i+5 .
\end{aligned}
$$

The labeling of $x_{0} y_{1}$ and $x_{1} y_{1}$ is fixed:

$$
\begin{aligned}
& f\left(x_{0} y_{1}\right)=1, \\
& f\left(x_{1} y_{1}\right)=13 .
\end{aligned}
$$

The square sum labeling of edge $x_{i} y_{i}$, for $i=(n / 2)$, is

$$
\begin{aligned}
f^{*}\left(x_{i} y_{i}\right) & =f\left(x_{i}\right)^{2}+f\left(y_{i}\right)^{2} \\
& =(2 n-1)^{2}+(2 n-2)^{2} \\
& =\left(4 n^{2}-4 n+1\right)+\left(4 n^{2}-8 n+4\right) \\
& =8 n^{2}-12 n+5 .
\end{aligned}
$$
is

The square sum labeling of $x_{i} y_{i}$, for $(n / 2)+1 \leq i \leq n-1$,

$$
\begin{aligned}
f^{*}\left(x_{i} y_{i}\right)= & f\left(x_{i}\right)^{2}+f\left(y_{i}\right)^{2} \\
= & \left((2 n-4)-4\left(i-\frac{n}{2}-1\right)\right)^{2} \\
& +\left((2 n-3-4)\left(i-\frac{n}{2}-1\right)\right)^{2} \\
= & 32 n^{2}-64 n i+32 i^{2}+8 n-8 i+1 .
\end{aligned}
$$

The square sum labeling of $y_{i} y_{i+1}$, for $2 \leq i \leq(n / 2)-2$, is 


$$
\begin{aligned}
f^{*}\left(y_{i} y_{i+1}\right) & =f\left(y_{i}\right)^{2}+f\left(y_{i+1}\right)^{2} \\
& =(4 i-2)^{2}+(4(i+1)-2)^{2} \\
& =\left(16 i^{2}-16 i+4\right)+\left(16 i^{2}+16 i+4\right) \\
& =32 i^{2}+8 .
\end{aligned}
$$

The following labels are fixed:

$$
\begin{aligned}
f^{*}\left(y_{0} y_{1}\right) & =5, \\
f^{*}\left(y_{1} y_{2}\right) & =40, \\
f^{*}\left(y_{n-1} y_{0}\right) & =26 .
\end{aligned}
$$

The square sum labeling of the edges $y_{i} y_{i+1}$, for $i=(n / 2)-1$, is

$$
\begin{aligned}
f^{*}\left(y_{i} y_{i+1}\right) & =f\left(y_{i}\right)^{2}+f\left(y_{i+1}\right)^{2} \\
& =(4 i-2)^{2}+(2 n-1)^{2} \\
& =16 i^{2}-16 i+4+4 n^{2}-4 n+1 .
\end{aligned}
$$

The labeling of the edges $y_{i} y_{i+1}$, for $i=(n / 2)$, is

$$
\begin{aligned}
f^{*}\left(y_{i} y_{i+1}\right) & =f\left(y_{i}\right)^{2}+f\left(y_{i+1}\right)^{2} \\
& =(2 n-1)^{2}+(2 n-3)^{2} \\
& =8 n^{2}-16 n+10 .
\end{aligned}
$$

The square sum labeling of the edges $y_{i} y_{i+1}$, for $(n / 2)+1 \leq i \leq n-2$, is

$$
\begin{aligned}
f^{*}\left(y_{i} y_{i+1}\right)= & f\left(y_{i}\right)^{2}+f\left(y_{i+1}\right)^{2} \\
= & \left((2 n-3)-4\left(i-\frac{n}{2}-1\right)\right)^{2} \\
& +\left((2 n-3)-4\left(i-\frac{n}{2}-1\right)\right)^{2} \\
= & 32 n^{2}-64 n i-16 n+32 i^{2}+16 i+10 .
\end{aligned}
$$

$$
\begin{aligned}
f^{*}\left(x_{i} x_{i+k}\right) & =f\left(x_{i}\right)^{2}+f\left(x_{i+k}\right)^{2} \\
& =\left(2 n-4-4\left(i-\frac{n}{2}-1\right)\right)^{2}+\left(2 n-4-4\left(i+k-\frac{n}{2}-1\right)\right)^{2} \\
& =\left(16 n^{2}-32 n i+16 i^{2}+16 i^{2}\right)+\left(16 n^{2}-32 n i-32 n k+32 i k+16 i^{2}+16 k^{2}\right) \\
& =32 n^{2}-64 n i+32 i^{2}+32 n k+16 i^{2}+16 k^{2} .
\end{aligned}
$$

The labeling of $x_{i} x_{i+k}$, for $(n / 2)+1 \leq i \leq n-1$ and $i+k=n=0 \bmod n$, is 


$$
\begin{aligned}
f^{*}\left(x_{i} x_{i+k}\right) & =f\left(x_{i}\right)^{2}+f\left(x_{i+k}\right)^{2} \\
& =\left(2 n-4-4\left(i-\frac{n}{2}-1\right)\right)^{2}+0 \\
& =16 n^{2}+16 i^{2}-32 n i-32 n k+32 i k+16 k^{2} .
\end{aligned}
$$

The labeling of $x_{i} x_{i+k}$, for $(n / 2)+1 \leq i \leq n-1$ and $i+$ $k>n$ and $($ say $i+k=j$ and $\bmod n), i \leq j \leq(n / 2)-2$, is

$$
\begin{aligned}
f^{*}\left(x_{i} x_{i+k}\right)= & f\left(x_{i}\right)^{2}+f\left(x_{i+k}\right)^{2} \\
= & \left(2 n-4-4\left(i-\frac{n}{2}-1\right)\right)^{2}+(4 j-1)^{2} \\
= & 16 n^{2}+16 i^{2}-32 n i-32 n k+32 i k+16 k^{2} \\
& +16 j^{2}-8 j+1 .
\end{aligned}
$$

From the computations of all the induced weights of the edges, we can easily see that they are distinct, so the graph is square sum for $n$ even.

\section{Case 2. When $n$ is odd.}

Suppose $G \cong P(n, k)$ be the generalized Petersen graph, where $2 \leq k \leq\lfloor n / 2\rfloor$ and $n$ is odd. We shall show that $G$ is square sum graph by defining a square sum labeling on $G$. So, let $\phi: V(G) \longrightarrow\{0,1, \ldots,|V(G)-1|\}$ be a function defined as follows.

The labeling of $\left(y_{n-1}\right),\left(y_{n-2}\right) \ldots\left(y_{n-6}\right)$ is given below:

$$
\begin{aligned}
& f\left(y_{n-1}\right)=2, f\left(y_{n-2}\right)=3, f\left(y_{n-3}\right)=5, \\
& f\left(y_{n-4}\right)=8, f\left(y_{n-5}\right)=10, f\left(y_{n-6}\right)=12 .
\end{aligned}
$$

These are the fixed numbers of the above labeling.

The labeling of vertices of $y_{0}$ is

$$
f\left(y_{0}\right)=2 n-1 \text {. }
$$

The labeling of vertices $y_{i}$ for $1 \leq i \leq(n / 2)$ is

$$
f\left(y_{i}\right)=2 n-1-2 i .
$$
below:

The labeling of vertices $x_{n-1}, x_{n-2} \ldots, x_{n-6}$ is given

$$
\begin{aligned}
& f\left(x_{n-1}\right)=1, \\
& f\left(x_{n-2}\right)=4, \\
& f\left(x_{n-3}\right)=6, \\
& f\left(x_{n-4}\right)=7, \\
& f\left(x_{n-5}\right)=9, \\
& f\left(x_{n-6}\right)=11 .
\end{aligned}
$$

These are the fixed numbers of the above labeling:

$$
f\left(x_{0}\right)=0 .
$$

The labeling $x_{i}$ for $1 \leq i \leq n-7$ is

$$
f\left(x_{i}\right)=2 n-2 i \text {. }
$$

This labeling is explained in Figure 2.

From all these computations of the weights of the edges, we can easily see that they are all distinct, that is, the induced labeling function $f^{*}$ is injective just the same way as in Case 1. This shows that $f$ is a square sum labeling, and therefore, $P(n, k)$ is a square sum graph.

2.1. Square Sum Labeling of Double Generalized Petersen Graph. The concept of double generalized Petersen graph was introduced by Zhou and Feng in 2012 (see [6]), where the automorphism group of these graph was characterized. Double generalized Petersen graph $\operatorname{DP}(n, k)$ is defined as the graph with vertex and edge set, as in Figure 3, particularly, for $k=1$.

In the next theorem, we have proved that the double generalized Petersen graph is a square sum graph for a particular case when $k=1$.

Theorem 2. Suppose $\mathrm{DP}(n, 1)$ be the double generalized Petersen graph; then, there exists square sum labeling of $D P(n, 1)$, for all $n \geq 5$.

Proof. Suppose $G \cong D P(n, 1)$ be the double generalized Petersen graph, where $0 \leq i \leq n-1$. We want to show that $G$ is a square sum graph by defining a square sum labeling on $G$. So, suppose $f: V(G) \longrightarrow\{0,1, \ldots,|V(G)-1|\}$ be a function defined as follows.

The labeling of $a_{i}$ vertices, for $0 \leq i \leq n-1$, is

$$
f\left(a_{i}\right)=4 i \text {. }
$$

The labeling of $b_{i}$, for $0 \leq i \leq n-1$, is

$$
f\left(b_{i}\right)=4 i+1 \text {. }
$$

The labeling of $c_{i}$, for $0 \leq i \leq n-1$, is

$$
f\left(c_{i}\right)=4 i+2 \text {. }
$$

The labeling of $d_{i}$, for $0 \leq i \leq n-1$, is

$$
f\left(d_{i}\right)=4 i=3 \text {. }
$$

This above labeling is explained in Figure 4.

Now, we shall discuss the weights of the edges induced by the above labeling of vertices of $G$. The weight of the edge $u v \in E(G)$ will be denoted as $f^{*}(u v)=(f(u))^{2}+(f(v))^{2}$. So, the induced labeling of the edge $a_{i} b_{i}$, for $0 \leq i \leq n-1$, is given as

$$
\begin{aligned}
f^{*}\left(a_{i} b_{i}\right) & =\left(f\left(a_{i}\right)\right)^{2}+\left(f\left(b_{i}\right)\right)^{2} \\
& =(4 i)^{2}+(4 i+1)^{2} \\
& =32 i^{2}+8 i+1 .
\end{aligned}
$$

The labeling of $c_{i} d_{i}$, for $0 \leq i \leq n-1$, is 


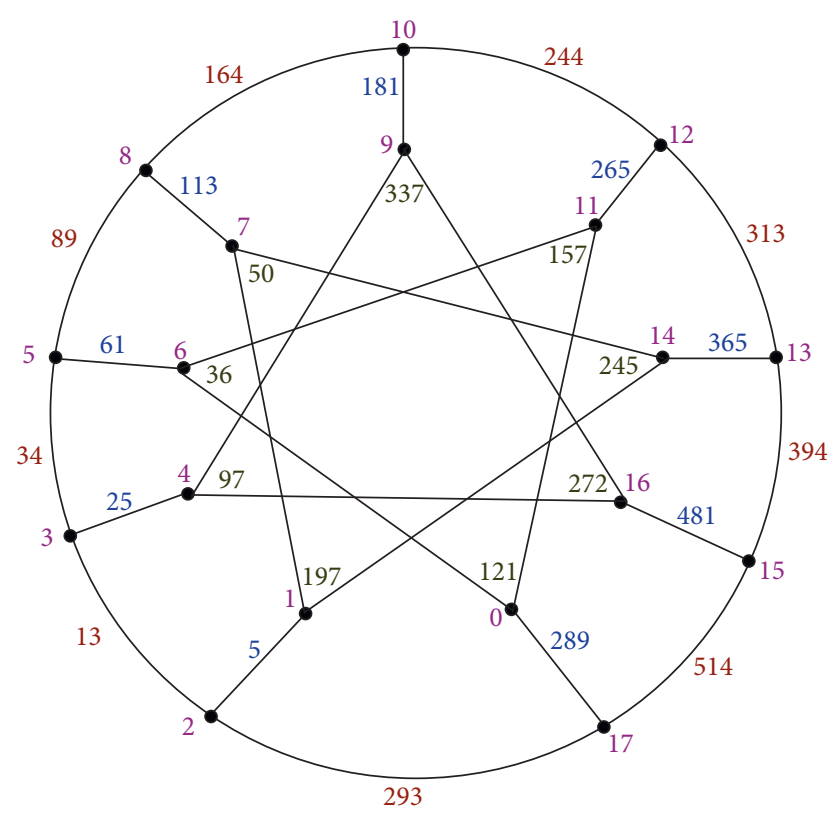

FIgURE 2: Square sum labeling of Petersen graph P [9,3].

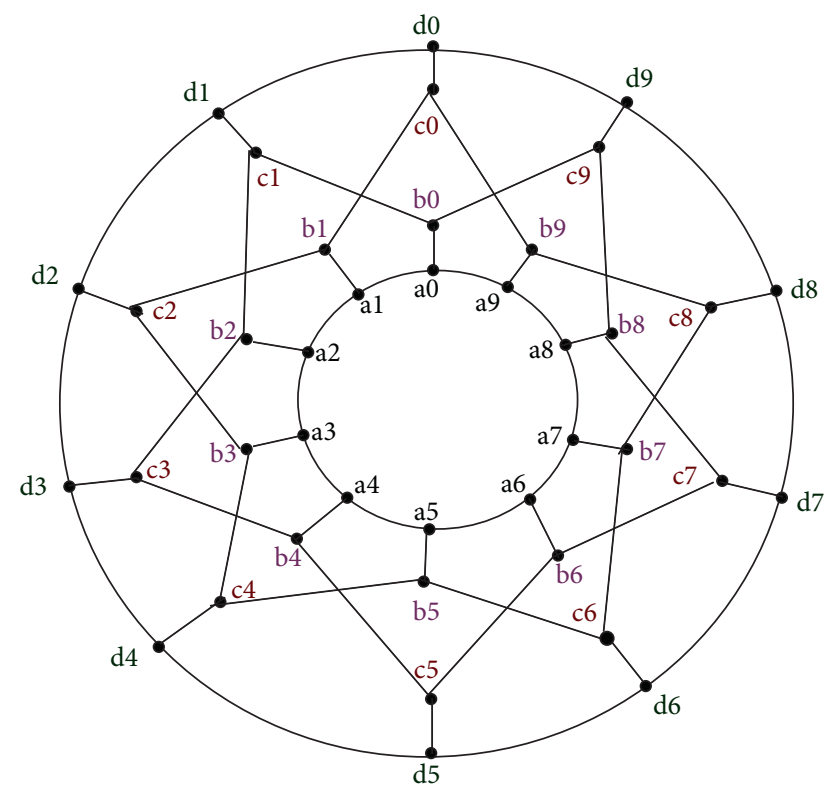

Figure 3: Generalized double Petersen graph $D P(10,1)$.

$$
\begin{aligned}
f^{*}\left(c_{i} d_{i}\right) & =f\left(c_{i}\right)^{2}+f\left(d_{i}\right)^{2} \\
& =(4 i+2)^{2}+(4 i+3)^{2} \\
& =32 i^{2}+40 i+13 .
\end{aligned}
$$

The labeling of $b_{i} c_{i+1}$, for $0 \leq i \leq n-2$, is

$$
\begin{aligned}
f^{*}\left(b_{i} c_{i+1}\right) & =f\left(b_{i}\right)^{2}+f\left(c_{i+1}\right)^{2} \\
& =(4 i+1)^{2}+(4(i+1)+2)^{2} \\
& =16 i^{2}+8 i+16(i+1)^{2}+16(i+1)+5 .
\end{aligned}
$$

The labeling of $a_{i} a_{i+1}$, for $0 \leq i \leq n-2$, is

$$
\begin{aligned}
f^{*}\left(a_{i} a_{i+1}\right) & =f\left(a_{i}\right)^{2}+f\left(a_{i+1}\right)^{2} \\
& =(4 i)^{2}+(4(i+1))^{2} \\
& =16 i^{2}+(4 i+4)^{2} \\
& =16 i^{2}+16 i^{2}+32 i+16 \\
& =32 i^{2}+32 i+16 .
\end{aligned}
$$

The labeling of $d_{i} d_{i+1}$, for $0 \leq i \leq n-2$, is

$$
\begin{aligned}
f^{*}\left(d_{i} d_{i+1}\right) & =f\left(d_{i}\right)^{2}+f\left(d_{i+1}\right)^{2} \\
& =(4 i+3)^{2}+(4(i+1)+3)^{2} \\
& =16 i^{2}+24 i+9+16 i^{2}+56 i+49 \\
& =32 i^{2}+80 i+58 .
\end{aligned}
$$

The labeling of the edges $b_{i} c_{i}$, for $0 \leq i \leq n-2$, is

$$
\begin{aligned}
f^{*}\left(b_{i} c_{i}\right) & =f\left(b_{i}\right)^{2}+f\left(c_{i}\right)^{2} \\
& =(4 i+1)^{2}+(4 i+2)^{2} \\
& =i=n-1, \quad i=0 \\
& =(4(n-1)+1)+(4(0)+2)^{2} \\
& =(4 n-4+1)^{2}+(4(0)+2)^{2} \\
& =16 n^{2}-24 n+13 .
\end{aligned}
$$

The labeling of the edges $b_{i+1} c_{i}$, for $0 \leq i \leq n-2$, is

$$
\begin{aligned}
f^{*}\left(b_{i+1} c_{i}\right) & =f\left(b_{i+1}\right)^{2}+f\left(c_{i}\right)^{2} \\
& =(4 i+1)^{2}+(4 i+2)^{2} \\
& =(4(i+1)+1)^{2}+(4 i+2)^{2} \\
& =((4 i+4)+1)^{2}+(4 i+2)^{2} \\
& =(4 i+4+1)^{2}+(4 i+2)^{2} \\
& =(4 i+5)^{2}+(4 i+2)^{2} \\
& =16 i^{2}+40 i+25+16 i^{2}+16 i+4 \\
& =32 i^{2}+56 i+29 .
\end{aligned}
$$

The labeling of the edges $a_{n-1} a_{0}$, for $0 \leq i \leq n-2$, is

$$
\begin{aligned}
f^{*}\left(a_{n-1} a_{0}\right) & =f\left(a_{n-1}\right)^{2}+f\left(a_{0}\right)^{2} \\
& =(4 i)^{2}+(4 i)^{2} \\
& =(4(n-1))^{2}+(4(0))^{2} \\
& =(4 n-4)^{2} \\
& =16 n^{2}+32 n+16 .
\end{aligned}
$$

The labeling of the edges $d_{n-1} d_{0}$, for $0 \leq i \leq n-2$, is 


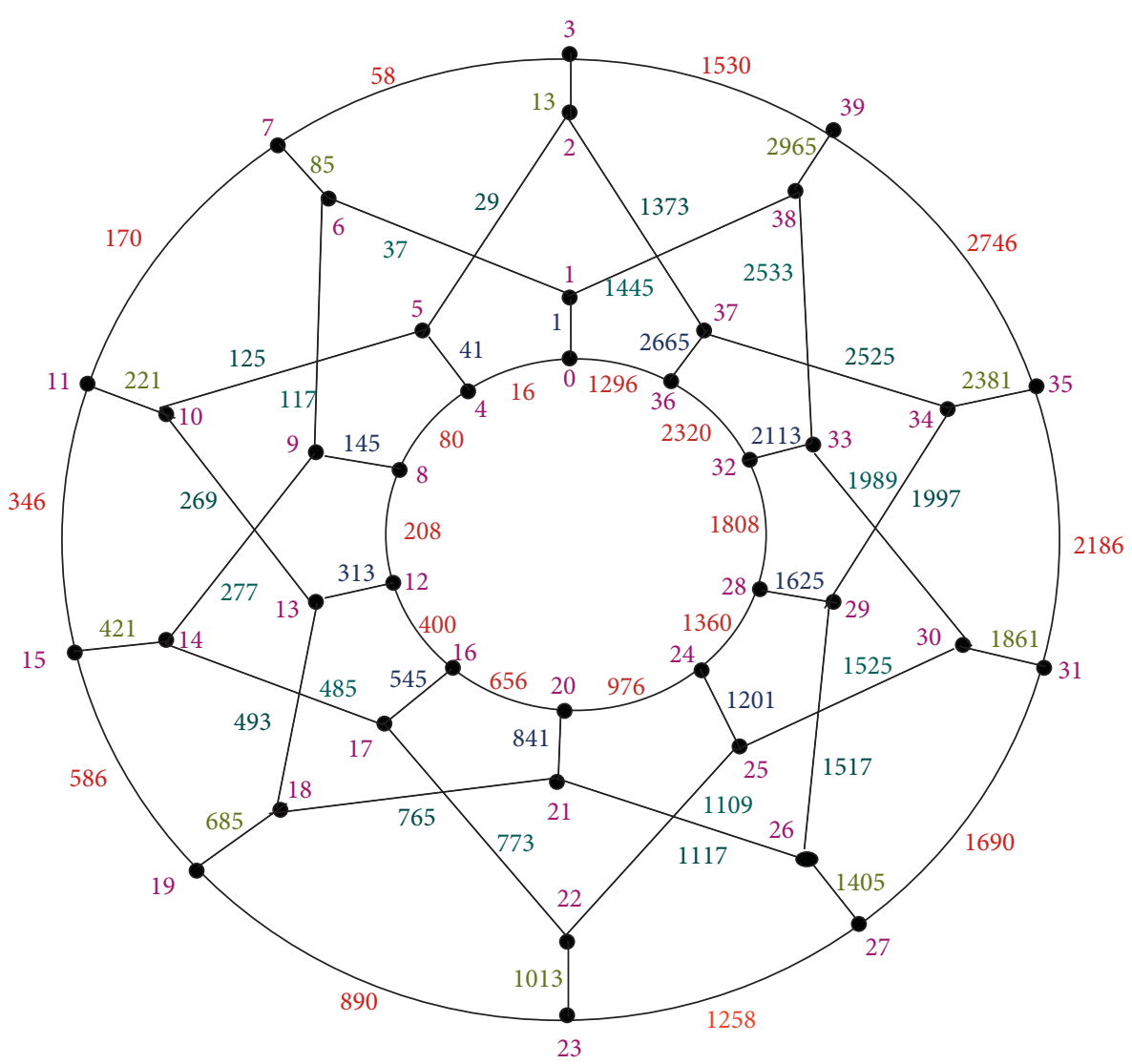

FIgURE 4: Square sum labeling of double generalized Petersen graph $D P[10,1]$.

$$
\begin{aligned}
f^{*}\left(d_{n-1} d_{0}\right) & =f\left(d_{n-1}\right)^{2}+f\left(d_{0}\right)^{2} \\
& =(4 i+3)^{2}+(4 i+3)^{2} \\
& =(4(n-1)+3)^{2}+(4(0)+3)^{2} \\
& =(4 n-4+3)^{2}+(4(0)+3)^{2} \\
& =(4 n-1)^{2}+(3)^{2} \\
& =16 n^{2}-8 n+10 .
\end{aligned}
$$

From all these computations of the weights of the edges, we can easily see that they are all distinct, that is, the induced labeling function $f^{*}$ is injective. This shows that $f$ is a square sum labeling, and therefore, $\operatorname{DP}(n, 1)$ is a square sum graph.

In the next theorem, we prove that "the double generalized Petersen graph $D P[n, 2]$ is a square sum graph for a particular case when $k=2$."

Theorem 3. Double generalized Petersen graph DP[n,2] is square sum for $n \geq 5$.

Proof. Let $G \cong D P(n, 2)$ be the double generalized Petersen graph. For this purpose, we shall consider two cases one is when $n$ is even and other $n$ is odd.
Case 3. When $n$ is even.

In this case, we define a labeling $f: V(G) \longrightarrow\{0,1,2,3 \ldots|V(G)|-1\}$, which will show that this labeling is a square sum labeling. Then, we discuss about the weights of the edges induced by the following labeling. The labeling of the outer edges:

$$
\begin{aligned}
f\left(a_{0}\right) & =0, \\
f\left(a_{n / 2}\right) & =1, \\
f\left(a_{n-1}\right) & =2, \\
f\left(a_{(n / 2)+1}\right) & =2 .
\end{aligned}
$$

The labeling of the vertices $a_{i}$, for $1 \leq i \leq(n / 2)$, is

$$
\begin{aligned}
f\left(a_{i}\right) & =2(i-1)+3, \\
f\left(a_{(n / 2)-1}\right) & =n-1 .
\end{aligned}
$$

The labeling of the vertices $a_{i}$, for $(n / 2)+2 \leq i \leq n-1$, is

$$
f\left(a_{i}\right)=n-2-2\left(i-\frac{n}{2}-1\right) .
$$

The labeling of inner vertices of $b_{i}^{\prime} s$ is 


$$
\begin{aligned}
f\left(b_{0}\right) & =n, \\
f\left(b_{n / 2}\right) & =n+1 .
\end{aligned}
$$

The labeling of the vertices $b_{i}$, for $1 \leq i \leq(n / 2)$, is

$$
\begin{aligned}
f\left(b_{i}\right) & =n+2(i-1)+3, \\
f\left(b_{(n / 2)+1}\right) & =2 n-2, \\
f\left(b_{(n / 2)-1}\right) & =2 n-2, \\
f\left(b_{(n / 2)+2}\right) & =2 n-4 .
\end{aligned}
$$

The labeling of the vertices $b_{i}$, for $(n / 2)+2 \leq i \leq n-1$, is

$$
\begin{aligned}
f\left(b_{i}\right) & =2 n-2-2\left(i-\frac{n}{2}-1\right), \\
f\left(b_{(n / 2)-2}\right) & =2 n-3 .
\end{aligned}
$$

The labeling of inner vertices of $c_{i^{\prime} s}$ is

$$
\begin{aligned}
f\left(c_{0}\right) & =2 n, \\
f\left(c_{n / 2}\right) & =2 n+1 .
\end{aligned}
$$

The labeling of $c_{i}$, for $1 \leq i \leq(n / 2)$, is

$$
\begin{aligned}
f\left(c_{i}\right) & =2 n+2(i-1)+3, \\
f\left(c_{n-1}\right) & =2 n+2, \\
f\left(c_{n-2}\right) & =2 n+4 \\
f\left(c_{(n / 2)-2}\right) & =3 n-3 \\
f\left(c_{(n / 2)-1}\right) & =3 n-1, \\
f\left(c_{(n / 2)+1}\right) & =3 n-2, \\
f\left(c_{(n / 2)+2}\right) & =3 n-4 .
\end{aligned}
$$

The labeling of $c_{i}$, for $(n / 2)+2 \leq i \leq n-1$, is

$$
f\left(c_{i}\right)=3 n-2-2\left(i-\frac{n}{2}-1\right) \text {. }
$$

The labeling of the inner vertices of $d_{i^{\prime} s}$ is

$$
\begin{aligned}
f\left(d_{0}\right) & =3 n, \\
f\left(d_{(n / 2)-1}\right) & =4 n-1, \\
f\left(d_{n-1}\right) & =3 n+2, \\
f\left(d_{1}\right) & =3 n+3 .
\end{aligned}
$$

The labeling of the vertices $d_{i}$, for $1 \leq i \leq(n / 2)$, is

$$
\begin{aligned}
f\left(d_{i}\right) & =3 n+2(i-1)+3, \\
f\left(d_{(n / 2)+1}\right) & =4 n-2, \\
f\left(d_{n / 2}\right) & =3 n+1 .
\end{aligned}
$$

The labeling of $d_{i}$, for $(n / 2)+2 \leq i \leq n-1$, is

$$
f\left(d_{i}\right)=4 n-2-2\left(i-\frac{n}{2}-1\right) \text {. }
$$

This labeling is explained in Figure 5.
The labeling of outer edges of $a_{i}$ and inner vertices of $b_{i}$, for $1 \leq i \leq n-1, i \neq 0, i \neq(n / 2)$, is

$$
\begin{aligned}
f^{*}\left(a_{i} b_{i}\right) & =f\left(a_{i}\right)^{2}+f\left(b_{i}\right)^{2} \\
& =(2(i-1)+3)^{2}+(n+2(i-1)+3)^{2} \\
& =8 i^{2}+8 i+n^{2}+4 i n+2 n+2 .
\end{aligned}
$$

The labeling of inner vertices of $c_{i}$ and inner edges of $b_{i}$, for $1 \leq i \leq n-1, i \neq 0, i=(n / 2)$, is

$$
\begin{aligned}
f^{*}\left(c_{i} d_{i}\right) & =f\left(c_{i}\right)^{2}+f\left(d_{i}\right)^{2} \\
& =(2 n+2(i-1)+3)^{2}+(3 n+2(i-1)+3)^{2} \\
& =13 n^{2}+20 i n+10 n+8 i^{2}+8 i+2, \\
f^{*}\left(c_{(n / 2)+1} d_{(n / 2)+1}\right) & =\left(f\left(c_{(n / 2)+1}\right)\right)^{2}+\left(f\left(d_{(n / 2)+1}\right)\right)^{2} \\
& =(3 n-2)^{2}+(4 n-2)^{2} \\
& =25 n^{2}-28 n+8 .
\end{aligned}
$$

The labeling of the edges $a_{i} b_{i}$, for $(n / 2)+2 \leq i \leq n-1$, is $f^{*}\left(a_{i} b_{i}\right)=\left(f\left(a_{i}\right)\right)^{2}+\left(f\left(b_{i}\right)\right)^{2}$

$$
\begin{aligned}
& =\left(n-2-2\left(i-\frac{n}{2}-1\right)\right)^{2}+\left(2 n-2-2\left(i-\frac{n}{2}-1\right)\right)^{2} \\
& =13 n^{2}-20 i n+8 i^{2} .
\end{aligned}
$$

The labeling of the edges $c_{i} d_{i}$, for $(n / 2)+2 \leq i \leq n-1$, is $f^{*}\left(c_{i} d_{i}\right)=\left(f\left(c_{i}\right)\right)^{2}+\left(f\left(d_{i}\right)\right)^{2}$

$$
\begin{aligned}
& =\left(3 n-2-2\left(i-\frac{n}{2}-1\right)\right)^{2}+\left(4 n-2-2\left(i-\frac{n}{2}-1\right)\right)^{2} \\
& =41 n^{2}-36 i n+8 i^{2} .
\end{aligned}
$$

The labeling of the edges $b_{i} c_{i+2}$, for $1 \leq i \leq(n / 2)$, is

$$
\begin{aligned}
f^{*}\left(b_{i} c_{i+2}\right) & =\left(f\left(b_{i}\right)\right)^{2}+\left(f\left(c_{i+2}\right)\right)^{2} \frac{n}{2}+1 \leq i \leq n-3 \\
& =(n+2(i-1)+3)^{2}+(2 n+2(i+2-1)+3)^{2} \\
& =5 n^{2}+8 i^{2}+12 i n+22 n+24 i+26 .
\end{aligned}
$$

The labeling of $c_{(n / 2)+1} b_{i+2}$ is given below:

$$
\begin{aligned}
f^{*}\left(c_{(n / 2)+1} b_{i+2}\right) & =\left(f\left(c_{(n / 2)}\right)\right)^{2}+\left(f\left(b_{i+2}\right)\right)^{2} \\
& =(3 n-2)^{2}+\left(2 n-2-2\left(i-\frac{n}{2}-1\right)\right)^{2} \\
& =(3 n-2)^{2}+\left(2 n-2-2\left(i+2-\frac{n}{2}-1\right)\right)^{2} \\
& =18 n^{2}+4 i^{2}-36 n+16 i-12 i n+20 .
\end{aligned}
$$




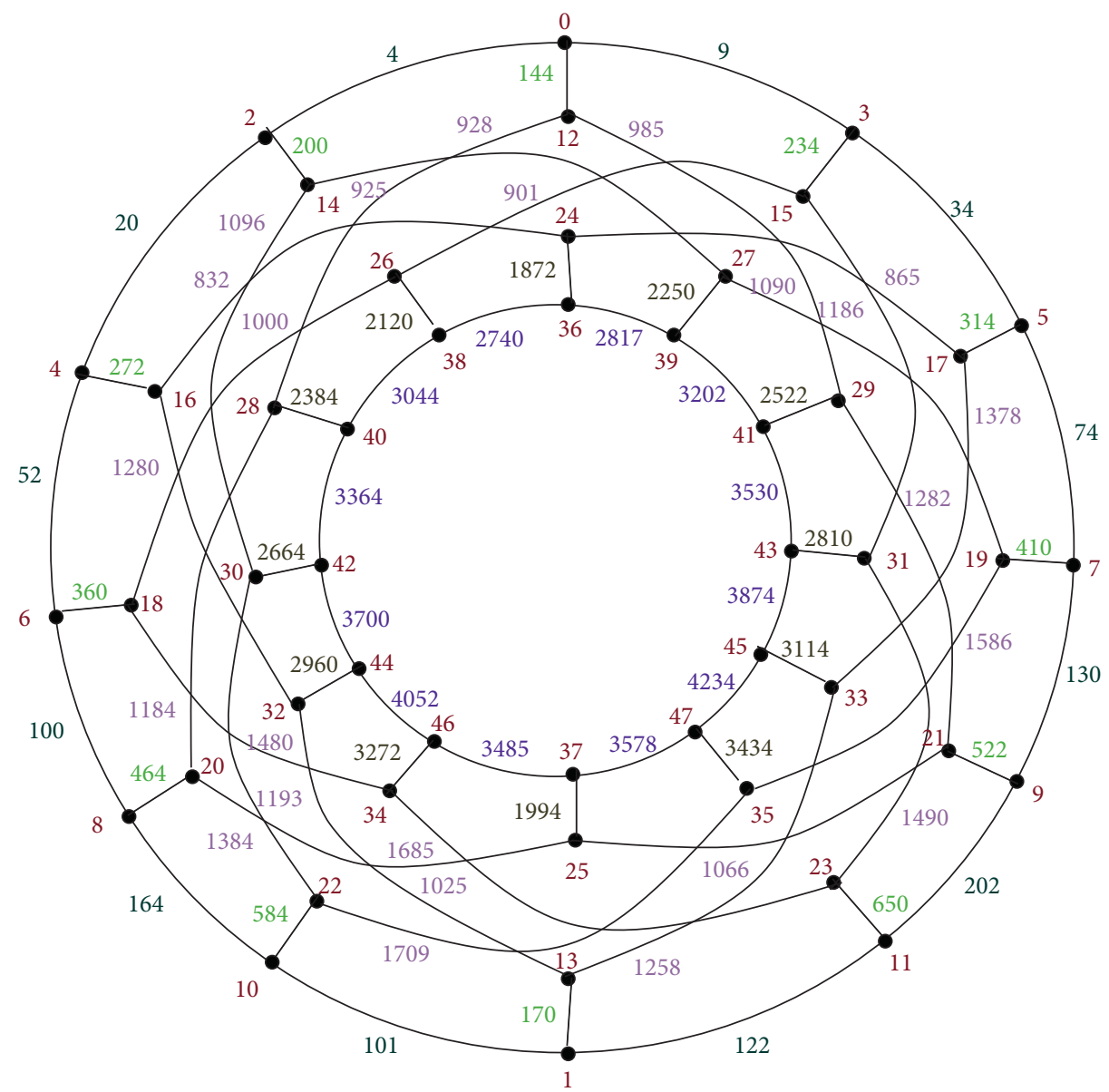

FIgUre 5: Square sum labeling of $D P[12,2]$.

The labeling of the edges $b_{i+2} c_{i}$, for $(n / 2)+1 \leq i \leq n-3$, is $f^{*}\left(b_{i+2} c_{i}\right)=\left(f\left(b_{i+2}\right)\right)^{2}+\left(f\left(c_{i}\right)\right)^{2}$

$$
\begin{aligned}
= & \left(2 n-2-2\left(i-\frac{n}{2}-1\right)\right)^{2} \\
& +\left(3 n-2-2\left(i-\frac{n}{2}-1\right)\right)^{2} \\
= & (3 n-2 i+4)^{2}+(4 n-2 i)^{2} \\
= & 25 n^{2}-28 i n-24 n+8 i^{2}+16 i+16 .
\end{aligned}
$$

The labeling of the edges $a_{0} b_{0}$ is given below:

$$
\begin{aligned}
f^{*}\left(a_{0} b_{0}\right) & =\left(f\left(a_{0}\right)\right)^{2}+\left(f\left(b_{0}\right)\right)^{2} \\
& =(0)^{2}+(n)^{2} \\
& =n^{2} .
\end{aligned}
$$

The labeling of the edges $a_{n / 2} b_{n / 2}$ is given below:

$$
\begin{aligned}
f^{*}\left(a_{n / 2} b_{n / 2}\right) & =\left(f\left(a_{n / 2}\right)\right)^{2}+\left(f\left(\frac{n}{2}\right)\right)^{2} \\
& =(1)^{2}+(n+1)^{2} \\
& =n^{2}+2 n+2 .
\end{aligned}
$$

The labeling of the edges $c_{0} d_{0}$ is given below:

$$
\begin{aligned}
f^{*}\left(c_{0} d_{0}\right) & =\left(f\left(c_{0}\right)\right)^{2}+\left(f\left(d_{0}\right)\right)^{2} \\
& =(2 n)^{2}+(3 n)^{2} \\
& =4 n^{2}+9 n^{2} \\
& =13 n^{2} .
\end{aligned}
$$

The labeling of edges $c_{n / 2} d_{n / 2}$ is given below:

$$
\begin{aligned}
f^{*}\left(c_{n / 2} d_{n / 2}\right) & =\left(f\left(c_{n / 2}\right)\right)^{2}+\left(f\left(d_{n / 2}\right)\right)^{2} \\
& =(2 n+1)^{2}+(3 n+1)^{2} \\
& =13 n^{2}+10 n+2 .
\end{aligned}
$$


The labeling of vertices, for $b_{(n / 2)-2} c_{n / 2}$, is given below:

$$
\begin{aligned}
f^{*}\left(b_{(n / 2)-2} c_{n / 2}\right) & =\left(f\left(b_{(n / 2)-2}\right)\right)^{2}+\left(f\left(c_{n / 2}\right)\right)^{2} \\
& =(2 n-3)^{2}+(2 n+1)^{2} \\
& =8 n^{2}-8 n+10 .
\end{aligned}
$$

The labeling of edges $b_{(n / 2)-2} c_{n / 2}$ is given below:

$$
\begin{aligned}
f^{*}\left(b_{(n / 2)-1} c_{(n / 2)+1}\right) & =\left(f\left(b_{(n / 2)-1}\right)\right)^{2}+\left(f\left(c_{(n / 2)+1}\right)\right)^{2} \\
& =(2 n-1)^{2}+(3 n-2)^{2} \\
& =13 n^{2}-16 n+5 .
\end{aligned}
$$
below:

The labeling of edges and vertices, for $b_{n / 2} c_{(n / 2)+2}$, is given

$$
\begin{aligned}
f^{*}\left(b_{n / 2} c_{(n / 2)+2}\right) & =\left(f\left(b_{n / 2}\right)\right)^{2}+\left(f\left(c_{(n / 2)+2}\right)\right)^{2} \\
& =(n+1)^{2}+(3 n-4)^{2} \\
& =10 n^{2}-22 n+17 .
\end{aligned}
$$

The labeling of vertices $b_{i} c_{i+2}$, for $(n / 2)+1 \leq i \leq n-3$, is

$$
\begin{aligned}
f^{*}\left(b_{i} c_{i+2}\right) & =\left(f\left(b_{i}\right)\right)^{2}+\left(f\left(c_{i+2}\right)\right)^{2} \\
& =(2 n-2)^{2}+\left(3 n-2-2\left(i-\frac{n}{2}-1\right)\right)^{2} \\
& =4 n^{2}-8 n+4+\left(3 n-2-2\left(i+2-\frac{n}{2}-1\right)\right)^{2} \\
& =4 n^{2}-8 n+4+(4 n-2 i-4)^{2} \\
& =20 n^{2}-40 n-16 i n+4 i^{2}+16 i+20 .
\end{aligned}
$$

The labeling of vertices $b_{n-2} c_{0}$ is given below:

$$
\begin{aligned}
f^{*}\left(b_{n-2} c_{0}\right) & =\left(f\left(b_{n-2}\right)\right)^{2}+\left(f\left(c_{o}\right)\right)^{2} \\
& =(n+4)^{2}+(2 n)^{2} \\
& =5 n^{2}+8 n+16 .
\end{aligned}
$$

The labeling of the edges $b_{n-1} c_{1}$ is given below:

$$
\begin{aligned}
f^{*}\left(b_{n-1} c_{1}\right) & =\left(f\left(b_{n-1}\right)\right)^{2}+\left(f\left(c_{1}\right)\right)^{2} \\
& =(n+2)^{2}+(2 n+2(i-1)+3)^{2}, \quad i=1 \\
& =n^{2}+4 n+4+(2 n+3)^{2} \\
& =5 n^{2}+16 n+13 .
\end{aligned}
$$

The labeling of the edges $b_{i+2} c_{i}$, for $1 \leq i \leq(n / 2)-3$, is

$$
\begin{aligned}
f^{*}\left(b_{i+2} c_{i}\right) & =\left(f\left(b_{i+2}\right)\right)^{2}+\left(f\left(c_{i}\right)\right)^{2} \\
& =(n+2(i-1)+3)^{2}+(2 n+2(i-1)+3)^{2} \\
& =(n+2 i+5)^{2}+(2 n+i+2)^{2} \\
& =5 n^{2}+5 i^{2}+8 i n+18 n+24 i+29 .
\end{aligned}
$$

The labeling of the edges $c_{0} b_{2}$ is given below:

$$
\begin{aligned}
f^{*}\left(c_{0} b_{2}\right) & =\left(f\left(c_{o}\right)\right)^{2}+\left(f\left(b_{2}\right)\right)^{2} \\
& =(2 n)^{2}+(n+2(i-1)+3)^{2} \\
& =(2 n)^{2}+(n+2(1)+3)^{2} \\
& =(2 n)^{2}+(n+5)^{2} \\
& =5 n^{2}+10 n+25 .
\end{aligned}
$$

The labeling of the vertices $c_{n-1} b_{1}$ is given below:

$$
\begin{aligned}
f^{*}\left(c_{n-1} b_{1}\right) & =\left(f\left(c_{n-1}\right)\right)^{2}+\left(f\left(b_{1}\right)\right)^{2} \\
& =(2 n+2)^{2}+(n+2(i-1)+3)^{2} \\
& =4 n^{2}+8 n+4+(n+3)^{2} \\
& =4 n^{2}+8 n+4+n^{2}+6 n+9 \\
& =5 n^{2}+14 n+13 .
\end{aligned}
$$

The labeling of the vertices $c_{n-2} b_{0}$ is given below:

$$
\begin{aligned}
f^{*}\left(c_{n-2} b_{0}\right) & =\left(f\left(c_{n-2}\right)\right)^{2}+\left(f\left(b_{0}\right)\right)^{2} \\
& =(2 n+4)^{2}+(n)^{2} \\
& =4 n^{2}+16 n+16+n^{2} \\
& =5 n^{2}+16 n+16 .
\end{aligned}
$$

The labeling of the edges $b_{n / 2} c_{(n / 2)-2}$ is given below:

$$
\begin{aligned}
f^{*}\left(b_{n / 2} c_{(n / 2)-2}\right) & =\left(f\left(b_{n / 2}\right)\right)^{2}+\left(f\left(c_{(n / 2)-2}\right)\right)^{2} \\
& =(n+1)^{2}+(3 n-3)^{2} \\
& =n^{2}+2 n+1+9 n^{2}-18 n+9 \\
& =10 n^{2}-16 n+10 .
\end{aligned}
$$

The labeling of the edges $b_{(n / 2)+2} c_{n / 2}$ is given below:

$$
\begin{aligned}
f^{*}\left(b_{(n / 2)+2} c_{n / 2}\right) & =\left(f\left(b_{(n / 2)+2}\right)\right)^{2}+\left(f\left(c_{n / 2}\right)\right)^{2} \\
& =(2 n-4)^{2}+(2 n+1)^{2} \\
& =8 n^{2}-12 n+17 .
\end{aligned}
$$

The labeling of the edges $b_{n / 2} c_{(n / 2)+2}$ is given here:

$$
\begin{aligned}
f^{*}\left(b_{n / 2} c_{(n / 2)-2}\right) & =\left(f\left(b_{n / 2}\right)\right)^{2}+\left(f\left(c_{(n / 2)-2}\right)\right)^{2} \\
& =(n+1)^{2}+(3 n-3)^{2} \\
& =n^{2}+2 n+1+9 n^{2}-18 n+9 \\
& =10 n^{2}-16 n+10 .
\end{aligned}
$$

The labeling of the edges $b_{(n / 2)+1} c_{(n / 2)-1}$ is given below:

$$
\begin{aligned}
f^{*}\left(b_{(n / 2)+1} c_{(n / 2)-1}\right) & =\left(f\left(b_{(n / 2)+1}\right)\right)^{2}+\left(f\left(c_{(n / 2)-1}\right)\right)^{2} \\
& =(2 n-2)^{2}+(3 n-1)^{2} \\
& =4 n^{2}-8 n+4+9 n^{2}-6 n+1 \\
& =13 n^{2}-14 n+5 .
\end{aligned}
$$


The labeling of the edges $a_{0} a_{1}$ is given below:

$$
\begin{aligned}
f^{*}\left(a_{0} a_{1}\right)^{2} & =\left(f\left(a_{0}\right)\right)^{2}+\left(f\left(a_{1}\right)\right)^{2} \\
& =(0)^{2}+(2(i-1)+3)^{2}, \quad i=1 \\
& =(0)^{2}+(2(0)+3)^{2} \\
& =(0)^{2}+(3)^{2} \\
& =9 .
\end{aligned}
$$

The labeling of the edges $a_{i} a_{i+1}$, for $1 \leq i \leq(n / 2)-2$, is

$$
\begin{aligned}
f^{*}\left(a_{i} a_{i+1}\right) & =\left(f\left(a_{i}\right)\right)^{2}+\left(f\left(a_{i+1}\right)\right)^{2} \\
& =(2(i-1)+3)^{2}+(2(i+1-1)+3)^{2} \\
& =(2 i+1)^{2}+(2 i+3)^{2} \\
& =8 i^{2}+16 i+10 .
\end{aligned}
$$

The labeling of the edges $a_{(n / 2)-1} a_{n / 2}$ is given below:

$$
\begin{aligned}
f^{*}\left(a_{(n / 2)-1} a_{n / 2}\right)^{2} & =\left(f\left(a_{(n / 2)-1}\right)\right)^{2}+\left(f\left(a_{n / 2}\right)\right)^{2} \\
& =(n-1)^{2}+(1)^{2} \\
& =n^{2}-2 n+2 .
\end{aligned}
$$

The labeling of edges with vertices having labels $a_{n / 2} a_{(n / 2)+1}$ is

$$
\begin{aligned}
f^{*}\left(a_{n / 2} a_{(n / 2)+1}\right) & =f\left(a_{n / 2}\right)^{2}+f\left(a_{(n / 2)+1}\right)^{2} \\
& =(1)^{2}+(n-2)^{2} \\
& =n^{2}-4 n+5 .
\end{aligned}
$$

The labeling of the edges $a_{n-1} a_{0}$ is here:

$$
\begin{aligned}
f^{*}\left(a_{n-1} a_{0}\right) & =\left(f\left(a_{n-1}\right)\right)^{2}+\left(f\left(a_{0}\right)\right)^{2} \\
& =(2)^{2}+(0)^{2} \\
& =4 .
\end{aligned}
$$

The square sum labeling of the edges $a_{i} a_{i+1}$, for $(n / 2)+2 \leq i \leq n-2$, is

$f^{*}\left(a_{i} a_{i+1}\right)=\left(f\left(a_{i}\right)\right)^{2}+f\left(a_{i+1}\right)^{2}, \quad \frac{n}{2}+2 \leq i \leq n-2$

$$
\begin{aligned}
= & \left(n-2-2\left(i-\frac{n}{2}-1\right)\right)^{2} \\
& +\left(n-2-2(i+1)-\frac{n}{2}-1\right)^{2} \\
= & (2 n-2 i)^{2}+(2 n-2 i-2)^{2} \\
= & 4 n^{2}-8 i n+4 i^{2}+4 n^{2}-8 i n-8 n+8 i+4 i^{2}+4 \\
= & 8 n^{2}-16 i n+8 i^{2}-8 n+8 i+4 .
\end{aligned}
$$

The labeling of $d_{0} d_{1}$ is the square sum:

$$
\begin{aligned}
f^{*}\left(d_{0} d_{1}\right) & =\left(f\left(d_{0}\right)\right)^{2}+\left(f\left(d_{1}\right)\right)^{2} \\
& =(3 n)^{2}+(3 n+3)^{2} \\
& =9 n^{2}+9 n^{2}+18 n+9 \\
& =18 n^{2}+18 n+9 .
\end{aligned}
$$

The labeling of the edges of $d_{0} d_{1}$ is the square sum:

$$
\begin{aligned}
f^{*}\left(d_{i} d_{i+1}\right) & =\left(f\left(d_{i}\right)\right)^{2}+\left(f\left(d_{i+1}\right)\right)^{2} \\
& =(3 n+2(i-1)+3)^{2}+(3 n+2(i+1-1)+3)^{2} \\
& =(3 n+2 i+1)^{2}+(3 n+2 i+3)^{2} \\
& =9 n^{2}+12 i n+18 n+4 i^{2}+12 i+9+9 n^{2}+12 i n+4^{2}+4 i+6 n+1 \\
& =18 n^{2}+24 i n+24 n+8 i^{2}+16 i+10 .
\end{aligned}
$$

The labeling of the edges $d_{(n / 2)-1} d_{n / 2}$ is given below:

$$
\begin{aligned}
f^{*}\left(d_{(n / 2)-1} d_{n / 2}\right) & =f\left(d_{(n / 2)-1}\right)^{2}+\left(f\left(d_{n / 2}\right)\right)^{2} \\
& =(4 n-1)^{2}+(3 n+1)^{2} \\
& =16 n^{2}-8 n+1+9 n^{2}+6 n+1 \\
& =25 n^{2}-2 n+2 .
\end{aligned}
$$

The labeling of the edges $d_{n / 2} d_{(n / 2)+1}$ is the square sum:

$$
\begin{aligned}
f^{*}\left(d_{n / 2} d_{n / 2+1}\right) & =\left(f\left(d_{n / 2}\right)\right)^{2}+\left(f\left(d_{(n / 2)+1}\right)\right)^{2} \\
& =(n+1)^{2}+(4 n-2)^{2} \\
& =9 n^{2}+6 n+1+16 n^{2}-16 n+5 \\
& =25 n^{2}-10 n+5 .
\end{aligned}
$$




$$
\begin{aligned}
f^{*}\left(d_{n-1} d_{0}\right) & =\left(f\left(d_{n-1}\right)\right)^{2}+\left(f\left(d_{0}\right)\right)^{2} \\
& =(3 n+2)^{2}+(3 n)^{2} \\
& =9 n^{2}+6 n+6 n+4+9 n^{2} \\
& =18 n^{2}+12 n+4 .
\end{aligned}
$$

The labeling of the edges $d_{i} d_{i+1}$ is the square sum:

$$
\begin{aligned}
f^{*}\left(d_{i} d_{i+1}\right) & =\left(f\left(d_{i}\right)\right)^{2}+\left(f\left(d_{i+1}\right)\right)^{2} \\
& =\left(4 n-2-2\left(i-\frac{n}{2}-1\right)\right)^{2}+\left(4 n-2-2\left(i+1-\frac{n}{2}-1\right)\right)^{2} \\
& =(5 n-2 i)^{2}+(5 n-2 i-2)^{2} \\
& =25 n^{2}-20 i n+4 i^{2}+25 n^{2}-20 i n-20 n+4 i^{2}+8 i+4 \\
& =50 n^{2}-40 i n+8 i^{2}+8 i-20 n+4 .
\end{aligned}
$$

From all these computations of the weights of the edges, we can easily see that they are all distinct, that is, the induced labeling function $f^{*}$ is injective. This shows that $f$ is a square sum labeling, and therefore, $\operatorname{DP}(n, 2)$ is a square sum graph.

Here, we prove Case 2, when $n$ is odd.

So, this graph shows square sum labeling. Let $G \cong D P(n, 2)$ be the double generalized Petersen graph, where $1 \leq i \leq n-1$. We shall show that $G$ is square sum graph by defining a square sum labeling on $G$.

Case 4. When $n$ is odd.

For this case, we define a labeling $f: V(G) \longrightarrow 0,1,2,3 \ldots|V(G)|-1$, which will show that this labeling is a square sum labeling.

The labeling of $a_{0}$ is

$$
f\left(a_{0}\right)=1 .
$$

The labeling of $b_{0}$ is

$$
f\left(b_{0}\right)=n+2 .
$$

The labeling of $c_{0}$ is

$$
f\left(c_{0}\right)=n+1 .
$$

The labeling of $d_{0}$ is

$$
f\left(d_{0}\right)=0 .
$$

The labeling of $a_{i}$, for $1 \leq i \leq n-1$, is

$$
f\left(a_{i}\right)=i+1 .
$$

The labeling of $b_{i}$, for $1 \leq i \leq n-1$, is

$$
f\left(b_{i}\right)=n+2+i .
$$

The labeling of $c_{i}$, for $2 \leq i \leq n-1$, is

$$
f\left(c_{i}\right)=3 n+i .
$$

The labeling of $d_{i}$, for $1 \leq i \leq n-1$, is

$$
f\left(d_{i}\right)=2 n+i+1 \text {. }
$$

The labeling in this case is explained in Figure 6.

The square sum labeling of the edge $a_{0} b_{0}$ is

$$
\begin{aligned}
f^{*}\left(a_{0} b_{0}\right) & =\left(f\left(a_{0}\right)\right)^{2}+\left(f\left(b_{0}\right)\right)^{2} \\
& =(1)^{2}+(n+2)^{2} \\
& =n^{2}+4 n+5 .
\end{aligned}
$$

The labeling of edges with vertices having labels $a_{i} b_{i}$, for $1 \leq i \leq n-1$, is

$$
\begin{aligned}
f^{*}\left(a_{i} b_{i}\right) & =\left(f\left(a_{i}\right)\right)^{2}+\left(f\left(b_{i}\right)\right)^{2} \\
& =(i+1)^{2}+(n+i+2)^{2} \\
& =n^{2}+2 i^{2}+6 i+4 n+2 i n+5 .
\end{aligned}
$$

The square sum labeling of the labels $c_{0} d_{0}$ is

$$
\begin{aligned}
f^{*}\left(c_{0} d_{0}\right) & =\left(f\left(c_{0}\right)\right)^{2}+\left(f\left(d_{0}\right)\right)^{2} \\
& =(n+1)^{2}+(0)^{2} \\
& =n^{2}+2 n+1 .
\end{aligned}
$$

The square sum labeling of the labels $c_{1} d_{i}$ is

$$
\begin{aligned}
f^{*}\left(c_{1} d_{i}\right) & =\left(f\left(c_{1}\right)\right)^{2}+\left(f\left(d_{i}\right)\right)^{2} \\
& =(3 n+1)^{2}+(2 n+i+1)^{2} \\
& =13 n^{2}+10 n+i^{2}+4 i n+2 i+2 .
\end{aligned}
$$

The square sum labeling of the labels $c_{i} d_{i}$, for $2 \leq i \leq n-1$, is 


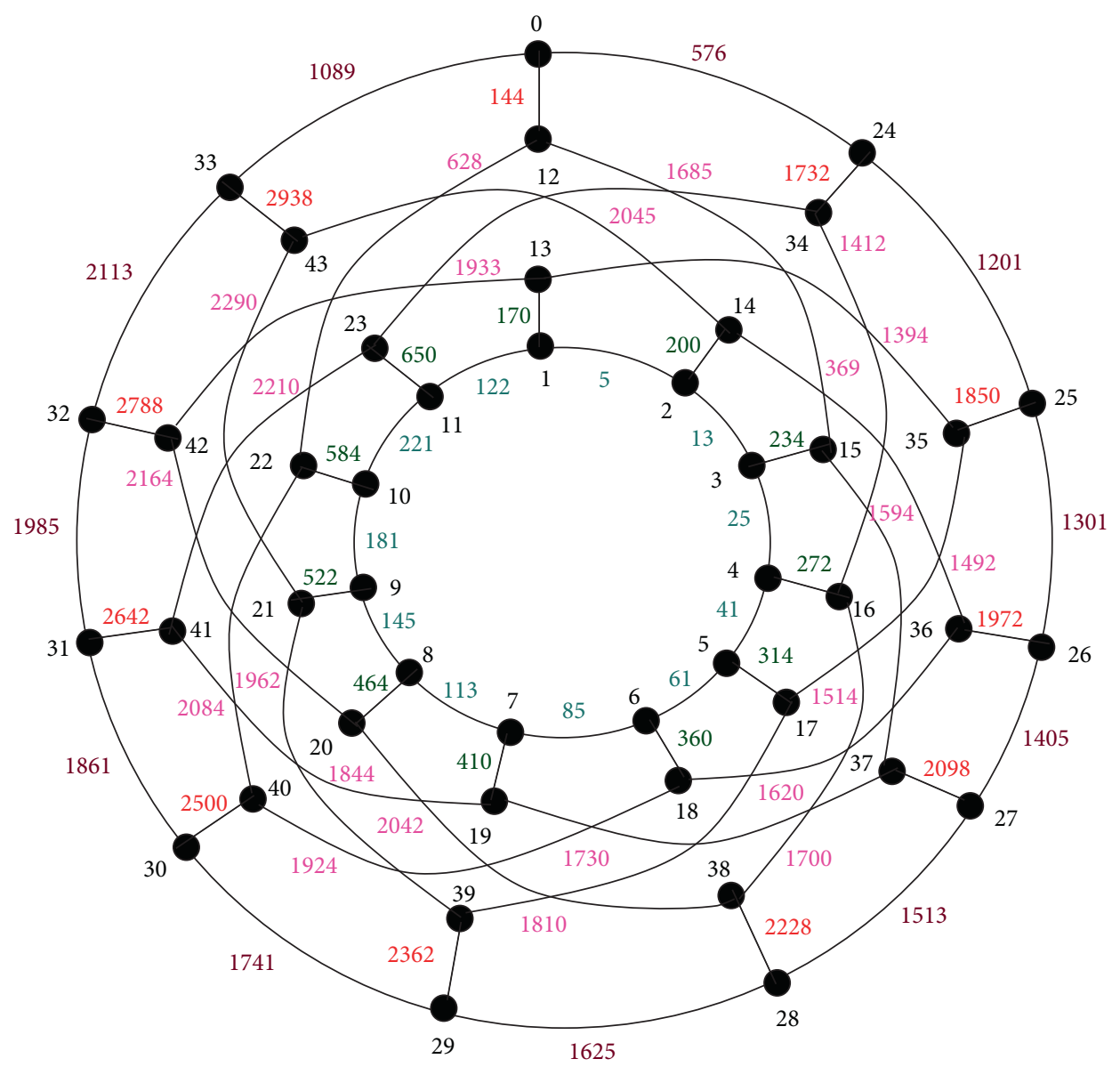

FIgURE 6: Square sum labeling of double generalized Petersen graph $[11,2]$.

$$
\begin{aligned}
f^{*}\left(c_{i} d_{i}\right) & =\left(f\left(c_{i}\right)\right)^{2}+\left(f\left(d_{i}\right)\right)^{2} \\
& =(3 n+i)^{2}+(2 n+i+1)^{2} \\
& =13 n^{2}+10 i n+2 i^{2}+4 n+2 i+1 .
\end{aligned}
$$

The square sum labeling of $d_{i} d_{0}$, for $i=n-1$, is

$$
\begin{aligned}
f^{*}\left(d_{i} d_{0}\right) & =\left(f\left(d_{i}\right)\right)^{2}+\left(f\left(d_{0}\right)\right)^{2} \\
& =(2 n+i+1)^{2}+(0)^{2} \\
& =(2 n+(n-1)+1)^{2}+(0)^{2} \\
& =(3 n)^{2} \\
& =9 .
\end{aligned}
$$

The square sum labeling of $a_{i} a_{0}$, for $i=n-1$, is

$$
\begin{aligned}
f^{*}\left(a_{i} a_{0}\right) & =\left(f\left(a_{i}\right)\right)^{2}+\left(f\left(a_{0}\right)\right)^{2} \\
& =(i+1)^{2}+(1)^{2} \\
& =((n-1)+1)^{2}+(1)^{2} \\
& =n^{2}+1^{2} \\
& =n^{2}+1 .
\end{aligned}
$$

The labeling of the edges $a_{i} a_{i+1}$, for $0 \leq i \leq n-2$, is given below:

$$
\begin{aligned}
f^{*}\left(a_{i} a_{i+1}\right) & =\left(f\left(a_{i}\right)\right)^{2}+\left(f\left(a_{i+1}\right)\right)^{2} \\
& =(i+1)^{2}+((i+1)+1)^{2} \\
& =(i+1)^{2}+(i+2)^{2} \\
& =2 i^{2}+6 i+5 .
\end{aligned}
$$
below:

The labeling of the edges $d_{i} d_{i+1}$, for $0 \leq i \leq n-2$, is given

$$
\begin{aligned}
f^{*}\left(d_{i} d_{i+1}\right) & =\left(f\left(d_{i}\right)\right)^{2}+\left(f\left(d_{i+1}\right)\right)^{2} \\
& =(2 n+i+1)^{2}+(2 n+(i+1)+1)^{2} \\
& =8 n^{2}+8 i n+12 n+2 i^{2}+6 i+5 .
\end{aligned}
$$

The square sum labeling of the edges $c_{0} b_{i}$, for $i=n-2$, is

$$
\begin{aligned}
f^{*}\left(c_{0} b_{i}\right) & =\left(f\left(c_{0}\right)\right)^{2}+\left(f\left(b_{i}\right)\right)^{2} \\
& =(n+1)^{2}+(n+i+2)^{2} \\
& =(n+1)^{2}+(n+2+(n-2))^{2} \\
& =(n+1)^{2}+(n+2+n-2)^{2}
\end{aligned}
$$




$$
\begin{aligned}
& =(n+1)^{2}+(2 n)^{2} \\
& =5 n^{2}+2 n+1 .
\end{aligned}
$$

The square sum labeling of the edges $b_{0} c_{i}$, for $i=n-2$, is

$$
\begin{aligned}
f^{*}\left(b_{0} c_{i}\right) & =\left(f\left(b_{0}\right)\right)^{2}+\left(f\left(c_{i}\right)\right)^{2} \\
& =(n+2)^{2}+(3 n+i)^{2} \\
& =(n+2)^{2}+(3 n+(n-2))^{2} \\
& =n^{2}+4 n+4+16 n^{2}+4-16 n \\
& =17 n^{2}-12 n+8 .
\end{aligned}
$$

The labeling of the edges $b_{i} c_{i}$ is

$$
\begin{aligned}
f^{*}=\left(b_{i} c_{i}\right) & =\left(f\left(b_{i}\right)\right)^{2}+\left(f\left(c_{i}\right)\right)^{2} \\
& =(n+2+i)^{2}+(3 n+i)^{2} \\
& =(n+2+1)^{2}+(3 n+i)^{2} ;\left(b_{i}\right)=n+2+i \Rightarrow i=1 \\
& =(n+3)^{2}+(3 n+(n-1))^{2} ;\left(c_{i}\right) \\
& =3 n+i \Rightarrow i=n-1 \\
& =(n+3)^{2}+(4 n-1)^{2} \\
& =17 n^{2}-2 n+10 .
\end{aligned}
$$

The square sum labeling of the edges $c_{0} b_{i}$, for $i=2$, is

$$
\begin{aligned}
f^{*}\left(c_{0} b_{i}\right) & =\left(f\left(c_{0}\right)\right)^{2}+\left(f\left(b_{i}\right)\right)^{2} \\
& =(n+1)^{2}+(n+i+2)^{2} \\
& =2 n^{2}+2 i n+6 n+i^{2}+4 i+5 .
\end{aligned}
$$

The square sum labeling of the edges $c_{i} b_{i+2}$, for $1 \leq i \leq n-3$, is

$$
\begin{aligned}
f^{*}\left(c_{i} b_{i+2}\right) & =\left(f\left(c_{i}\right)\right)^{2}+\left(f\left(b_{i+2}\right)\right)^{2} \\
& =(3 n+i)^{2}+(n+2+i)^{2} \\
& =10 n^{2}+8 n+8 i n+2 i^{2}+8 i+16 .
\end{aligned}
$$
is

The square sum labeling of the edges $c_{i} b_{i}$, for $0 \leq i \leq n-3$,

$$
\begin{aligned}
f^{*}\left(c_{i} b_{i}\right) & =\left(f\left(c_{i}\right)\right)^{2}+\left(f\left(b_{i}\right)\right)^{2} \\
& =(3 n+i)^{2}+(n+2+i)^{2} ;\left(c_{i}\right) \Rightarrow i=i+2 \\
& =(3 n+i+2)^{2}+(n+2+i)^{2} \\
& =10 n^{2}+8 i n+16 n+2 i^{2}+8 i+8 .
\end{aligned}
$$
is

The square sum labeling of the edges $c_{i} b_{i}$, for $0 \leq i \leq n-3$,

$$
\begin{aligned}
f^{*}\left(c_{i} b_{i}\right) & =\left(f\left(c_{i}\right)\right)^{2}+\left(f\left(b_{i}\right)\right)^{2} \\
& =(3 n+i)^{2}+(n+2+i)^{2} ;\left(c_{i}\right) \Rightarrow i=i+2 \\
& =(3 n+i+2)^{2}+(n+2+i)^{2} \\
& =10 n^{2}+8 i n+16 n+2 i^{2}+8 i+8 .
\end{aligned}
$$

The labeling of the edges $c_{1} b_{i}$ is given below:

$$
\begin{aligned}
f^{*}\left(c_{1} b_{i}\right) & =\left(f\left(c_{1}\right)\right)^{2}+\left(f\left(b_{i}\right)\right)^{2} \\
& =(3 n+1)^{2}+(n+2+i)^{2} ;\left(c_{i}\right) \Rightarrow i=n-1 \\
& =(3 n+1)^{2}+(n+2+(n-1))^{2} \\
& =(3 n+1)^{2}+(2 n+1)^{2} \\
& =13 n^{2}+10 n+2 .
\end{aligned}
$$

From all these computations of the weights of the edges, we can easily see that they are all distinct, that is, the induced labeling function $f^{*}$ is injective. This shows that $f$ is a square sum labeling, and therefore, $\operatorname{DP}(n, 2)$ is a square sum graph.

\section{Conclusion}

In this study, we have studied the square sum labeling of Generalized Petersen graph and double Generalized Petersen graph, and we prove that these graphs are square sum graphs.

\section{Data Availability}

All the data used to support the findings of the study are available within the article.

\section{Conflicts of Interest}

The authors declare that they have no conflicts of interest.

\section{Acknowledgments}

This work was supported by the National Key Research and Development Program, under Grant 2018YFB0904205.

\section{References}

[1] A. K. Germina, S. Arumugam, and V. Ajitha, "On square sum graphs," AKCE International Journal of Graphs and Combinatorics, vol. 6, no. 1, pp. 1-10, 2009.

[2] M. A. Seoud and M. N. Al-Harere, "Further results on square sum graphs," National Academy Science Letters, vol. 37, no. 5, pp. 473-475, 2014.

[3] M. I. Huilgol and V. Sriram, "Square sum labeling of disjoint union of graphs," Journal of Graph Labeling, vol. 2, no. 2, pp. 103-106, 2016.

[4] A. K. Germina and R. Sebastian, "On square sum graphs," Proyecciones, vol. 32, no. 2, pp. 107-117, 2013. 
[5] M. Watkins, "A theorem on tait colorings with an application to the generalized Petersen graphs," Journal of Combinatorial Theory, vol. 6, no. 2, pp. 152-164, 1969.

[6] J. X. Zhou and Y. Q. Feng, "Cubic vertex-transitive non-Cayley graphs of order 8p," The Electronic Journal of Combinatorics, vol. 19, no. 1, 2012. 